\title{
FINALLY A DOUBLE-MODE RR LYRAE MODEL?
}

\author{
J.R. Buchler and G. Kovács \\ University of Florida
}

ABSTRACT

It is shown that in a certain range of physical parameters a proper choice of the artificial viscosity parameters can lead to double-mode pulsation of RR Lyrae models. Here we present one such model and exhibit its double-mode behavior both through the stability analysis of the periodic limit cycles and through straightforward numerical integrations.

The lingering question of the cause of the observed double-mode pulsation of Cepheids, $\delta$ Scuti and RR Lyrae stars has remained unanswered since the first serious attempt of stellingwerf $(1975 \mathrm{a}, \mathrm{b})$ to find such models. Though stellingwerf was able to demonstrate that some of his cooler RR Lyrae models do pulsate in two modes simultaneously (when his special inner boundary conditions are applied), none of the subsequent studies of models with $\mathrm{f}$ iner envelope structure and $\mathrm{f}$ ixed inner boundary conditions were able to reproduce his results nor did they lead to any other double-mode models (see e.g., Cox, 1982).

Because the modal selection properties of a stellar model might be very sensitive to the many parameters (number of mass zones, viscosity, boundary conditions, et.c.) of the nonlinear model, we decided to perform a detailed nonlinear pulsational survey of many (50-100) RR Lyrae models. We used a slightly modified version of stellingwerf's (1975a) code with 60 mass zones down to the fixed inner boundary at 10-15\% of the stellar radius. We also took a finer sampling of the hydrogen ionization zone (20 zones of equal mass up to 15,000K). A detailed description of our models will be published elsewhere (Kovács and Buchler, 1986); here we summarize only briefly our main conclusions about the-double-mode RR Lyrae problem:

1. Models with the observed periods of double-mode RR Lyrae stars do not have simultaneously unstable fundamental and first overtone pulsations (which is a necessary condition for double-mode models).

2. Models with:

a. 2:1 resonance between the fundamental and third overtone, and low effective temperature

or:

b. roughly equal linear fundamental and first overtone growth rates close to the fundamental blue edge can show double mode pulsation for some specific range of the artificial viscosity parameters.

3. A better treatment of shocks and the inclusion of convection seems inevitable to produce double-mode models with period closer to the observed ones.

It is interesting to note that the possibility of double-mode pulsation due to a $2: 1$ resonance (case a.) has already been predicted by Dziembowski and Kovács (1984) and similarily, nonresonant double-mode pulsation (case b.) as a'general explanation of the observed double-mode stars was anticipated by Buchler and Kovács (1986). 


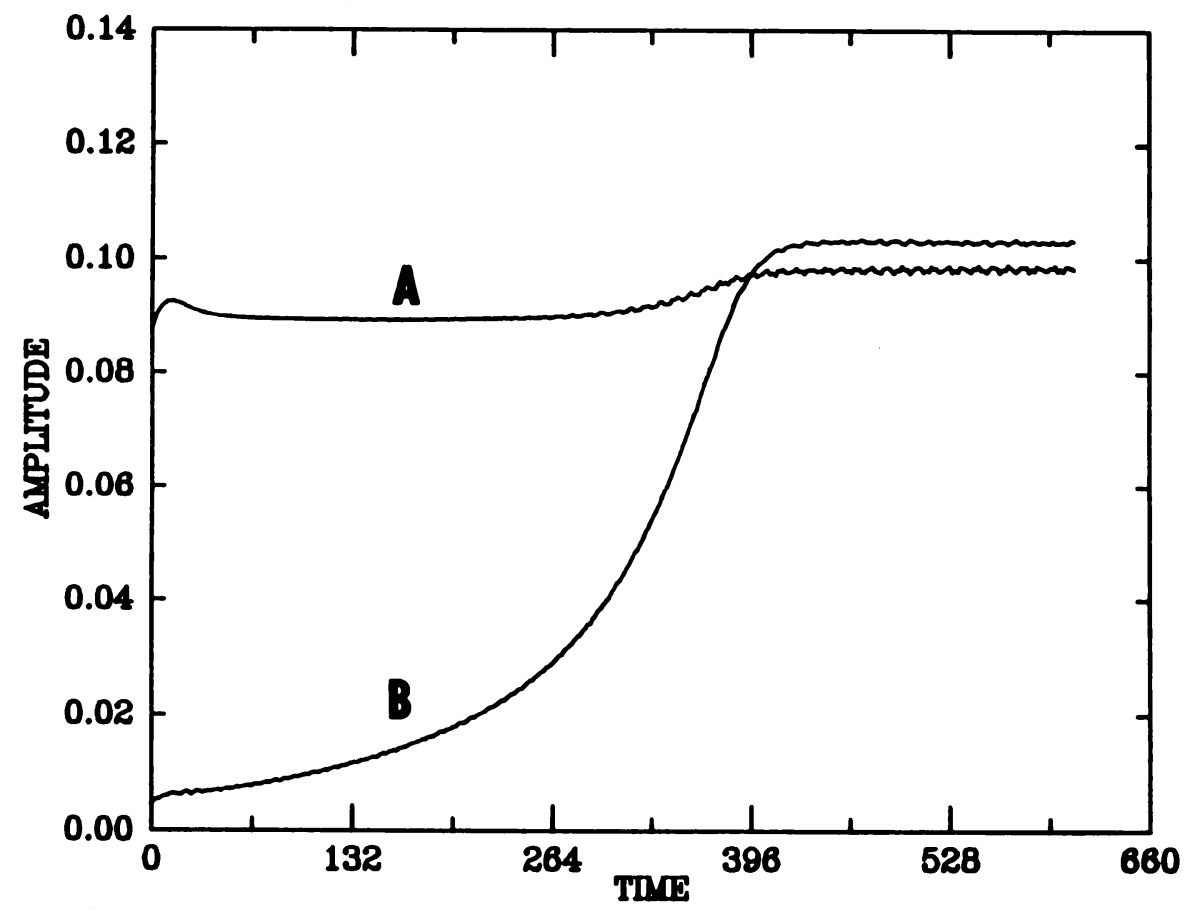

As an example we show the evolution of the principal amplitudes of the radius variation of one of our resonant models in Fig. 1. The model has the following parameters: $M=0.85 M_{\supset}, L=35 L_{\rho}$, Tef $f=6200, X=0.700$, $Y=0.299, C Q=20.0, a=0.03, P_{0}=0.438$ day, $P_{1} / P_{0}=0.758$. The model was initiated with a pure fundamental (linear) velocity distribution scaled to $10 \mathrm{~km} / \mathrm{s}$ at the surface, and then integrated up to 1400 fundamental periods. We denote the fundamental and first overtone amplitudes by $A$ and $B$, respectively (measured in solar units). Time is given in days. Other direct integrations of the model from different initial conditions also showed convergence to the same double-mode state. Furthermore, a linear stability analysis of the fundamental and first overtone limit cycles showed that they were unstable (the growth rate of the first overtone in the fundamental is 0.0052 , that of the fundamental in the first overtone is 0.0006 ).

This work has been supported by NSF AST84-10361, by the NCSA at the University of Illinois and by the NER Data Center at the University of Florida.

\section{REFERENCES}

Buchler, J.R. and Kovács, G. 1986, Ap. J. (Sep. 15).

Cox, A.N. 1982, Pulsation in Classical and Cataclismic Variable Stars, p. 157. Eds., Cox, J.P. and Hansen, C.J.

Dzi embowski, W. and Kovács, G. 1984, Mon. Not. Roy. Astr. Soc. 206, 497.

Kovács, G. and Buchler, J.R. 1986, Ap. J. (Submitted).

Stell ingwerf, R.F. 1975a, Ap.'J. 195, 441.

Stellingwerf, R.F. 1976a, Ap. J. $\underline{199}, 705$. 